\title{
Multiscale Entropy of Cardiac and Postural Control Reflects a Flexible Adaptation to a Cognitive Task
}

\author{
Estelle Blons ${ }^{1, * \mathbb{C}}$, Laurent M. Arsac ${ }^{1}$, Pierre Gilfriche ${ }^{1,2}$ and Veronique Deschodt-Arsac ${ }^{1}$ \\ 1 Univ. Bordeaux, CNRS, Laboratoire IMS, UMR 5218, 33400 Talence, France; \\ laurent.arsac@u-bordeaux.fr (L.M.A.); pierre.gilfriche@u-bordeaux.fr (P.G.); \\ veronique.arsac@u-bordeaux.fr (V.D.-A.) \\ 2 CATIE-Centre Aquitain des Technologies de l'Information et Electroniques, 33400 Talence, France \\ * Correspondence: estelle.blons@u-bordeaux.fr
}

Received: 16 September 2019; Accepted: 19 October 2019; Published: 21 October 2019

\begin{abstract}
In humans, physiological systems involved in maintaining stable conditions for health and well-being are complex, encompassing multiple interactions within and between system components. This complexity is mirrored in the temporal structure of the variability of output signals. Entropy has been recognized as a good marker of systems complexity, notably when calculated from heart rate and postural dynamics. A degraded entropy is generally associated with frailty, aging, impairments or diseases. In contrast, high entropy has been associated with the elevated capacity to adjust to an ever-changing environment, but the link is unknown between entropy and the capacity to cope with cognitive tasks in a healthy young to middle-aged population. Here, we addressed classic markers (time and frequency domains) and refined composite multiscale entropy (MSE) markers (after pre-processing) of heart rate and postural sway time series in 34 participants during quiet versus cognitive task conditions. Recordings lasted $10 \mathrm{~min}$ for heart rate and $51.2 \mathrm{~s}$ for upright standing, providing time series lengths of 500-600 and 2048 samples, respectively. The main finding was that entropy increased during cognitive tasks. This highlights the possible links between our entropy measures and the systems complexity that probably facilitates a control remodeling and a flexible adaptability in our healthy participants. We conclude that entropy is a reliable marker of neurophysiological complexity and adaptability in autonomic and somatic systems.
\end{abstract}

Keywords: heart rate variability; posture; entropy; complexity; cognitive task

\section{Introduction}

Physiological control is critical for health and well-being in humans, as it contributes to maintaining homeostasis and the adoption of adequate behaviors. Effective control takes place across intricate networks spanning many neural structures and operating across many time scales. These networks are dynamically organized to respond to internal and external stimuli. The coordinate functioning of the many constitutive components, their multiple interactions within and between systems, and the presence of overlapping control loops have promoted the conceptualization of nonlinear systems, exhibiting complexity [1].

The emergent field of systems physiology exploits the idea that complexity is mirrored in the temporal structure of a system's output variable. By analyzing physiological time series generated by control systems (e.g., the autonomic control of heart rate [1,2] or the somatic control of postural sway when standing upright $[3,4])$, researchers have discovered a preserved richness of the information carried by the output signals across multiple timescales. This richness in physiological signals can be assessed based on sample entropy [5], a measure of the irregularity of a time series obtained by calculating the probability that segments (also called vectors) of similar $m$ samples remain similar when 
the segment length increases to $m+1$. Entropy-based complexity metrics relate to the information content of a signal by quantifying the degree of regularity or predictability over one or more scales of time. To address this issue, Costa et al. [1,2] have proposed a multiscale entropy (MSE) method that consists of a coarse-graining process and sample entropy computations to measure the complexity of a time series at different temporal scales.

The true strength of this method lies in considering the sample entropy value over multiple time scales rather than one unique scale. By considering many scales, one can evaluate how far a system deviates both from emitting white noise (meaning a degraded network organization) and emitting a very regular signal, which is interpreted as too strict an organization and a lack of flexibility.

In agreement with these interpretations, experimental applications have demonstrated a degraded entropy in cardiac and postural dynamics associated with frailty, aging, impairments, or diseases $[1,3,4,6-19]$. By contrast, high entropy is generally associated with an elevated capacity to adjust to an ever-changing environment [8], and elevated values are often observed in young healthy people [1].

During a dual-task protocol, the degradation of entropy in postural sway is exacerbated in aged people $[3,8]$, thus indicating a failure in the dynamic re-organization of control. A similar phenomenon was observed in cardiovascular control when comparing nocturnal and diurnal MSEs of heart rate dynamics [1]. During waking periods, complexity raised in young individuals but vanished in old-age individuals, which lets the authors suppose that environmental stimuli (and the need for multi-tasking) may exceed a system's capacity, thus prohibiting an adequate re-organization in aged people.

One can ask whether stimuli not exceeding a system's capacity leads to an adequate re-organization of physiological control, and whether this is reflected in a greater signal entropy. In other words, it is unclear to date if the capacity to cope with a cognitive task in a healthy young to middle-age population is reflected in the entropy of a control system's output, while a degraded entropy seems to be the rule among old-aged individuals.

The aim of the present study is to assess the dynamic organization of control when performing cognitive tasks using the temporal behavior of heart rate and postural dynamics according to a multiscale entropy approach. We hypothesized that entropy would increase during the cognitive tasks, thus highlighting a flexible adaptation of neurophysiological control in our healthy participants.

\section{Materials and Methods}

\subsection{Population}

Thirty-four volunteers ( 8 women, 26 men) gave their written informed consent to participate in the present study in accordance with a local institutional review board policy and with the principles of the Declaration of Helsinki. The mean and standard deviation values of participants' age and body mass indexes were $30.5 \pm 14.0$ years (range: $18-59$ ) and $21.1 \pm 1.9 \mathrm{~kg} / \mathrm{m}^{2}$, respectively. Among the women, four were using oral contraceptives, five reported being in the follicular phase of their menstrual cycle and three were in the luteal phase. All volunteers had a university education.

None of the participants reported neurological or physiological disorders. Participants were asked to avoid alcohol and caffeinated beverages for the $12 \mathrm{~h}$ preceding the experiment, but also to abstain from heavy physical activity.

\subsection{Protocol}

The experimental protocol included recordings of heart rate dynamics and postural dynamics, according to reference (Ref) and cognitive tasks (Cog). Recordings of heart rate dynamics lasted 10 min during which the participants were sitting down in a quiet environment, breathing normally (at a spontaneous rate), and either facing a blank computer screen (Ref), or performing cognitive tasks displayed on the screen ( $\mathrm{Cog}$ ). Recordings of postural dynamics lasted $51.2 \mathrm{~s}$, during which the participants had to stand upright on a force platform, either looking at a black cross $4 \mathrm{~m}$ ahead 
(Ref), or performing a cognitive task displayed on a screen $4 \mathrm{~m}$ ahead (Cog). This study followed a randomized crossover design in which participants executed either cardiac or postural measurements first, and, in each of these two blocks of measurements, either Ref or Cog was executed first.

\subsection{Recordings of RR Interval Time Series}

Cardiac interbeat (RR interval) time series were recorded from a bipolar electrode transmitter belt Polar H7 (Polar, Finland) fitted to the chest of the subject and connected to an iPod (Apple, Cupertino CA, USA) via Bluetooth. A smartphone application was used to continuously store the transmitted RR intervals. About 500-600 successive RR intervals were recorded over $10 \mathrm{~min}$, the exact length of the RR interval time series depending on the average heart rate of each participant. For further analyses, the RR interval time series were exported to Matlab (Matworks, Natick, MA, USA).

\subsection{Recordings of Center of Pressure Time Series}

Anteroposterior (AP) and mediolateral (ML) postural sway was assessed from the center of pressure (COP) trajectory and recorded by a platform equipped with three strain gauges (Winposturo, Medicapteurs, $40 \mathrm{~Hz} / 16 \mathrm{~b}$, Balma, France). Participants stood barefoot with feet abducted at $15^{\circ}$ from the median line and heels separated by $4 \mathrm{~cm}$. Participants' eyes were open and their arms hung loosely at their sides. COP trajectories were recorded at a sampling frequency of $40 \mathrm{~Hz}$ for $51.2 \mathrm{~s}$ (thus providing 2048 data points). The AP and ML time series were exported to Matlab (Matlab R2017b, Mathworks) for further analyses.

\subsection{Cognitive Tasks}

During Cog, participants performed cognitive tasks chosen to solicit frontal cortical lobes, cerebral areas where executive functions operate [20,21].

During the entire 10-min recordings of heart rate dynamics, participants performed four tests that followed one another in this order: the Stroop Color and Word Test (SCWT) [22], the Hayling Sentence Completion Test (HSCT) [23], a visual version of the Paced Auditory Serial Addition Test (PASAT) [24], and a semantic fluency task [25]. In order to ensure that participants remained silent during these tests, they wrote their answers to the test. The durations of each task were the following: $3 \mathrm{~min}$ for the SCWT, $2.5 \mathrm{~min}$ for the HSCT, 3 min for the PASAT, and $1.5 \mathrm{~min}$ for the semantic fluency task. SCWT is a task that forces inhibition of cognitive interference, which occurs when the processing of a stimulus feature affects the simultaneous processing of another attribute of the same stimulus [22]. The HSCT taps into response initiation and response inhibition [23]. The PASAT requires attentional functioning, working memory, and information processing speed [24]. The semantic fluency task consisted of spontaneous narration about a given topic (e.g., supermarkets) [25].

Due to the short duration of the recordings of postural dynamics (51.2 s), SCWT alone was administrated. Participants answered verbally.

\subsection{Analysis of RR Interval Time Series: Classic Indices}

Due to technical issues, two participants (one woman and one man) were excluded from the RR interval time series analyses. All computations were performed in Matlab using available functions and custom-designed routines. The raw data of heart rate variability (HRV; RR interval time series) were inspected for artifacts. Occasional ectopic beats (irregularity of the heart rhythm involving extra or skipped heartbeats such as extrasystole and consecutive compensatory pause), were visually identified and manually replaced with interpolated adjacent RR interval values. Classic indices were then calculated in time and frequency domains. The mean of RR interval values was calculated. The root mean square of successive differences (RMSSD) was obtained by calculating the first difference, a discrete analog of the first derivative, which is a standard method for removing slow varying trends in a signal and highlights the power of high-frequencies that are associated with parasympathetic modulations of the heart rate [26]. In the frequency domain, discrete Fourier transform was performed 
after $4 \mathrm{~Hz}$ resampling using a cubic spline interpolation. The computation of signal power in fixed bands between 0.04 and $0.15 \mathrm{~Hz}$ for the low frequencies (LFs) and between 0.15 and $0.4 \mathrm{~Hz}$ for the high frequencies (HFs), allowed the calculation of the ratio LF/HF (an index of the sympathovagal balance) [26].

\subsection{Analysis of Center of Pressure Time Series: Classic Indices}

To evaluate the main features of postural control, here we computed the $95 \%$ confidence ellipse area, which is expected to enclose approximately $95 \%$ of the points on the COP path [19]. As well, the average velocity along the AP and the ML axes was computed. In the frequency domain, the spectral energy was assessed on ML and AP axes based on the power spectral density (PSD) obtained with fast Fourier transform.

\subsection{Analysis of Complexity: Entropy Indices}

The refined composite multiscale entropy (RCMSE) [27] was computed from both RR interval time series and postural time series in order to investigate signal complexity. As mentioned by Wu et al., the RCMSE method proposes improve the MSE method for short time series [2,27] by increasing the accuracy of entropy estimation and reducing the probability of inducing undefined entropy [27]. Undefined entropy may result from computations of short time series where no template segments (vectors) are matched to one another.

In brief, in the original MSE algorithm [1,2], the analyzed time series $x=\left\{x_{1}, x_{2}, \ldots, x_{N}\right\}$ is coarse grained using non-overlapping windows to obtain the representation of the original time series at different time scales $\tau$. The algorithm detects how many segments (vectors) of size $m$ remain similar at size $m+1$ in the time series. Hence, the number of matched vector pairs indicates the level of signal regularity. Due to a reduction of the original signal by a factor of $\tau$, the time series at large scale factors is composed of much fewer data points that the original one $[27,28]$. This is a concern for the accuracy of entropy calculation, mainly in short time series. A first attempt to address this accuracy concern was the development of composite multiscale entropy (CMSE) [29], whose main gain relies on considering all possible starting points at a given scale for the coarse-grained process, then calculating the averaged sample entropy for each scale. It was observed that CMSE, despite possessing a greater accuracy, increases the probability of inducing undefined entropy. To address this particular concern, Wu et al. (2014) [27] developed refined composite multiscale entropy (RCMSE), a method that uses the number of matched vector pairs for each scale factor $\tau$ and also for all $(k) \tau$ coarse-grained time series. Hence, it is unlikely even for short time series that the sum of matched vector pairs are zeros.

Briefly, the RCMSE algorithm consists of the following procedures (see detailed method in [27]):

1. At each scale factor of $\tau$, the number of matched vector pairs $n_{k, \tau}^{m+1}$ and $n_{k, \tau}^{m}$ is calculated for all $(k) \tau$ coarse-grained series, with $m$ corresponding to the sequence length considered. In the present study, $m=2$.

2. The RCMSE at a scale factor of $\tau$ is provided as follows, with $r$ corresponding to the tolerance for matches. In the present study, $r=0.15$ of the standard deviation of the initial time series $x$ [30].

$$
\operatorname{RCMSE}(x, \tau, m, r)=-\ln \left(\frac{\sum_{k=1}^{\tau} n_{k, \tau}^{m+1}}{\sum_{k=1}^{\tau} n_{k, \tau}^{m}}\right)
$$

The length of the original time series determines the largest analyzed scale $[1,27,31]$. In this study, RCMSE was assessed over a range of scales from 1 to 4 for RR interval time series and over a range of scales from 1 to 14 for postural times series, a difference that was due to different sample sizes of RR interval (500 to 600 samples) and postural (2048 samples) times series.

The RCMSE curve is obtained by plotting entropy values for each coarse-grained time series as a function of scales. The cardiac entropy index $\left(E_{C}\right)$ and postural entropy index $\left(E_{P}\right)$ are the area 
under the corresponding RCMSE curves (areas calculated using the trapezoidal rule) (Figure 1) [1,27]. As recommended by Gow et al. [31], entropy indices were computed after pre-processing time series using empirical mode decomposition (EMD) [32]. EMD decomposes a signal into a sum of intrinsic mode functions (IMFs) and a residual trend. This residual trend was subtracted to remove the drift, which has been identified as a source of error in entropy assessments [31].
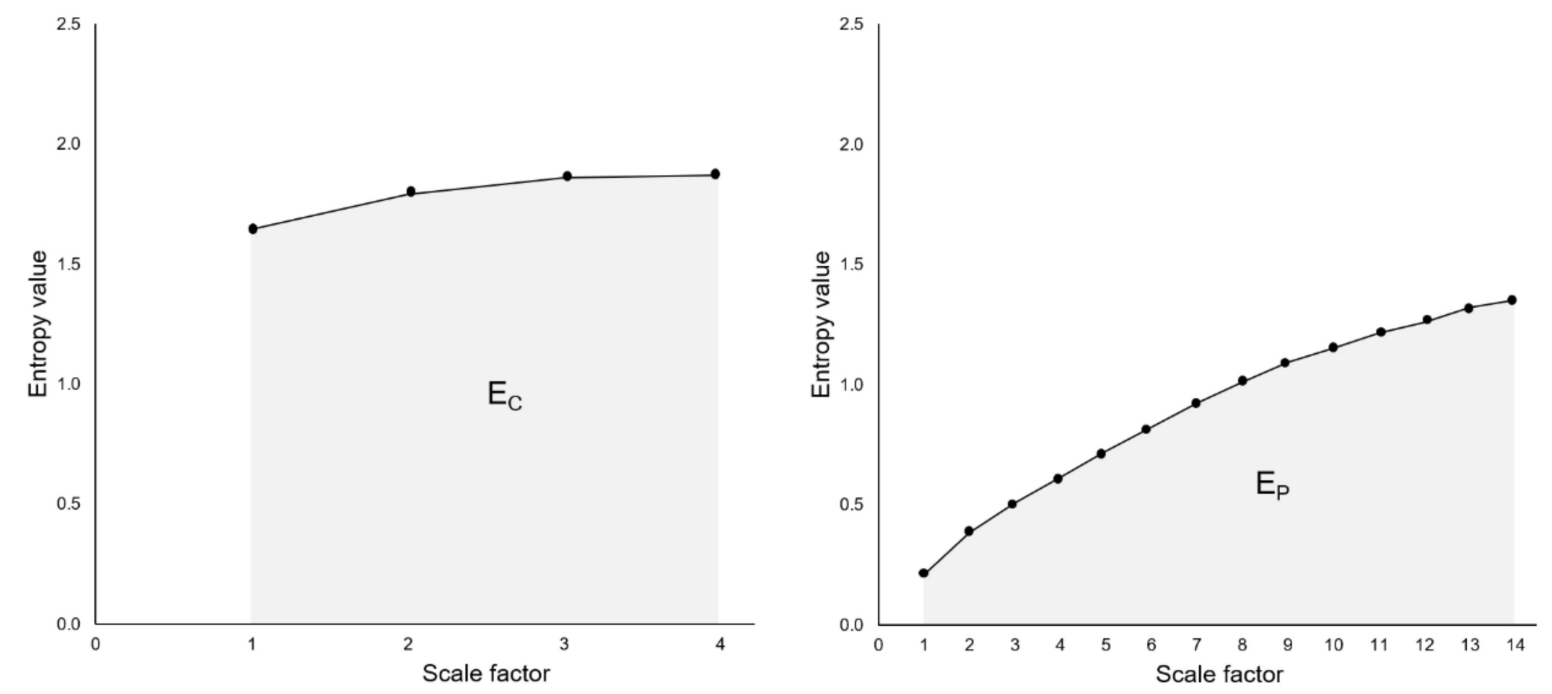

Figure 1. Cardiac entropy index $\left(\mathrm{E}_{\mathrm{C}}\right.$, left $)$ and postural entropy index $\left(\mathrm{E}_{\mathrm{P}}\right.$, right), calculated from the areas under the refined composite multiscale entropy (RCMSE) curves.

We tested the hypothesis that the complexity of our time series is encoded in the sequential ordering, and that this ordering is not fortuitous. For that, we built surrogate time series by shuffling the sequence of data points (randomly reordering). RCMSE curves are presented comparatively (see the figure in Section 3.2).

\subsection{Statistical Analyses}

All statistical procedures were conducted by use of XLSTAT (Addinsoft, 2019, XLSTAT statistical and data analysis solution, Long Island, NY, USA). Classic and entropy indices were tested for normality (Shapiro-Wilk test). These indices were compared between Ref and Cog conditions (two-tail $t$-test or Wilcoxon test). Following the American Statistical Association statement on statistical significance and $p$-values, we did not base our scientific conclusions only on whether a $p$-value passes a specific threshold (usually, $p<0.05$ ). Measures of detection sensitivity theory were additionally employed to assess sensitivity and specificity of the obtained indices, including the receiver operating characteristic (ROC) [33]. The area under the ROC curve indicates the probability that the index will assign a higher value to a positive instance than to a negative one [34]. Youden's index $(J=$ Sensitivity + Specificity -1$)$ assesses the performance of the detector.

\section{Results}

Figure 2 shows typical signal outputs from the two explored neurophysiological systems obtained for a single participant: RR interval times series under reference (Ref) and cognitive (Cog) conditions are shown in the top panel; anteroposterior (AP) and mediolateral (ML) time series of the COP trajectory are reported below in middle and bottom panels respectively.

Mean values of classic and entropy indices derived from the signals obtained from our participants are reported in Table 1. 
Ref
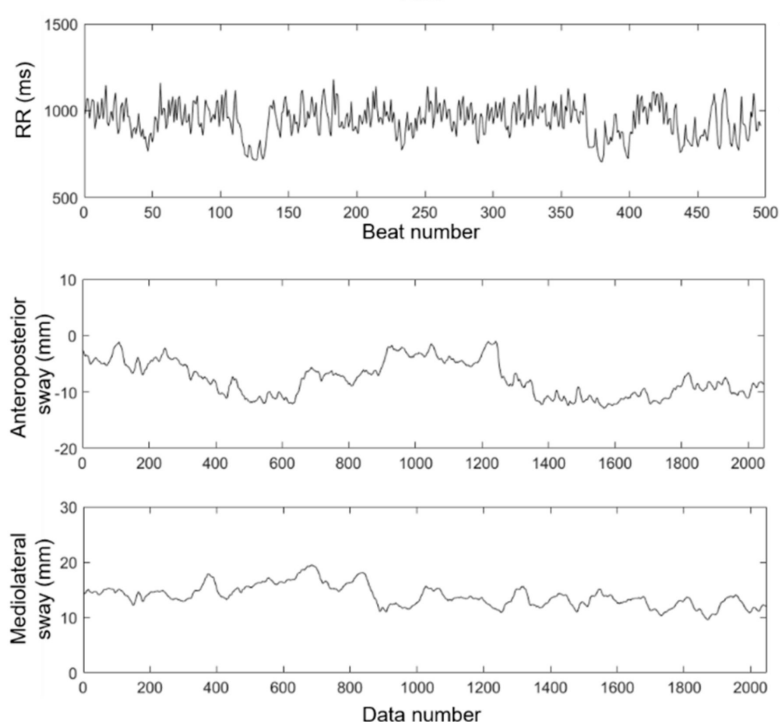

$\operatorname{Cog}$
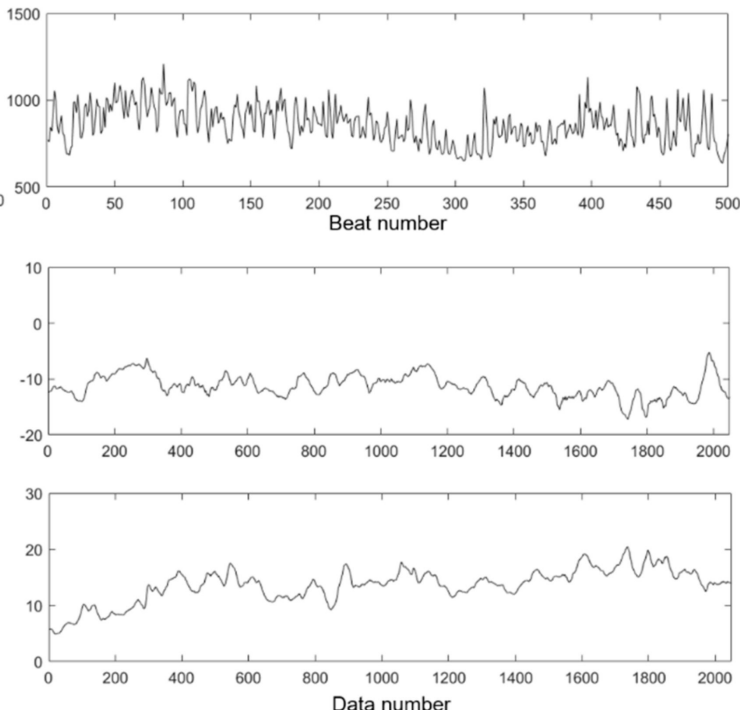

Figure 2. Top: RR interval time series from a representative participant in reference (Ref, left) and cognitive (Cog, right) conditions. Middle and bottom: anteroposterior (AP, middle) and mediolateral (ML, bottom) center of pressure (COP) time series, the horizontal axes are the same for these plots.

Table 1. Classic and entropy indices calculated from RR interval time series and from anteroposterior and mediolateral center of pressure time series, during reference and cognitive conditions.

\begin{tabular}{|c|c|c|}
\hline Heart Rate Dynamics & Ref & $\operatorname{Cog}$ \\
\hline RR intervals (ms) & $952 \pm 120$ & $915 \pm 131^{* *}$ \\
\hline RMSSD (ms) & $58 \pm 36$ & $52 \pm 30$ \\
\hline $\mathrm{LFs}\left(\mathrm{ms}^{2}\right)$ & $2243 \pm 2058$ & $1894 \pm 1602$ \\
\hline $\mathrm{HFs}\left(\mathrm{ms}^{2}\right)$ & $1459 \pm 1448$ & $1150 \pm 1196$ \\
\hline $\mathrm{LFs} / \mathrm{HFs}^{\prime}$ & $2.96 \pm 3.09$ & $2.82 \pm 2.62$ \\
\hline $\mathrm{E}_{\mathrm{C}}$ & $5.45 \pm 0.60$ & $5.75 \pm 0.69^{*}$ \\
\hline Postural Dynamics & Ref & $\operatorname{Cog}$ \\
\hline $95 \%$ confidence ellipse $\left(\mathrm{mm}^{2}\right)$ & $217.5 \pm 148.5$ & $184.7 \pm 103.5$ \\
\hline AP velocity $\left(\mathrm{mm} \cdot \mathrm{s}^{-1}\right)$ & $4.4 \pm 1.1$ & $5.1 \pm 1.2^{* * *}$ \\
\hline AP energy $\left(\mathrm{mm}^{2}\right)$ & $10.29 \pm 19.1$ & $9.04 \pm 5.5$ \\
\hline $\mathrm{AP} \mathrm{E}_{\mathrm{P}}$ & $11.81 \pm 3.07$ & $14.45 \pm 3.27^{* * *}$ \\
\hline ML velocity $\left(\mathrm{mm} \cdot \mathrm{s}^{-1}\right)$ & $4.9 \pm 1.6$ & $5.3 \pm 1.5^{*}$ \\
\hline ML energy $\left(\mathrm{mm}^{2}\right)$ & $6.42 \pm 3.59$ & $8.00 \pm 5.54$ \\
\hline ML E & $13.99 \pm 2.76$ & $14.72 \pm 3.03$ \\
\hline
\end{tabular}

Values provided are mean \pm standard deviation. Ref: reference condition; Cog: cognitive condition; RMSSD: root mean square of successive differences; LFs: low frequencies; HFs: high frequencies; $\mathrm{E}_{\mathrm{C}}$ : cardiac entropy index; AP: anteroposterior; $\mathrm{E}_{\mathrm{P}}$ : postural entropy index; ML: mediolateral. Differences between Ref and Cog are expressed as ${ }^{* * *} p<0.001,{ }^{* *} p<0.01,{ }^{*} p<0.05$.

\subsection{Classic Indices in Temporal and Frequency Domains}

The mean RR decreased (heart rate increased) under the Cog conditions ( $p<0.001$, two-tail Wilcoxon test).

None of the classic temporal (RMSSD) or frequency-derived heart rate variability (HRV) indices (LF, HF, LF/HF) differed between Ref and Cog, meaning that power at any given frequency did not change during Cog. Regarding posture, no difference in 95\% confidence ellipse or total PSD-derived energy was observed in the COP displacement signals, while the COP velocity differed (AP $p<0.001$, two-tail Wilcoxon test and ML $p=0.046$, two-tail Wilcoxon test). 


\subsection{Entropy Indices}

As expected, the RCMSE curves for the shuffled (randomly ordered) time series markedly differed from the RCMSE curves for the original time series (Figure 3). Entropy as a function of scales exhibited a monotonic decrease in shuffled time series, which is characteristic of random (white) noise [1,3]. By contrast, heart rate and postural dynamics exhibited typical behavior of a complex system, where the richness of carried information (as represented by entropy at a given scale) do not vanish when observed in longer timescales.

The main entropy index values $\left(\mathrm{E}_{\mathrm{C}}\right.$ and $\left.\mathrm{E}_{\mathrm{P}}\right)$ are presented in Table 1. As a main finding here, the $\mathrm{E}_{\mathrm{C}}$ index obtained during $\operatorname{Cog}$ was higher than the index obtained during $\operatorname{Ref}(p=0.016$, two-tail Wilcoxon test).

As well, along the AP axis where most of the postural (dys)regulation occurs [35,36], the $\mathrm{E}_{\mathrm{P}}$ index obtained during $\operatorname{Cog}$ was higher than the index obtained during $\operatorname{Ref}(p<0.001$, two-tail t-test). The ML $\mathrm{E}_{\mathrm{P}}$ indices did not differ between Ref and Cog (Table 1).
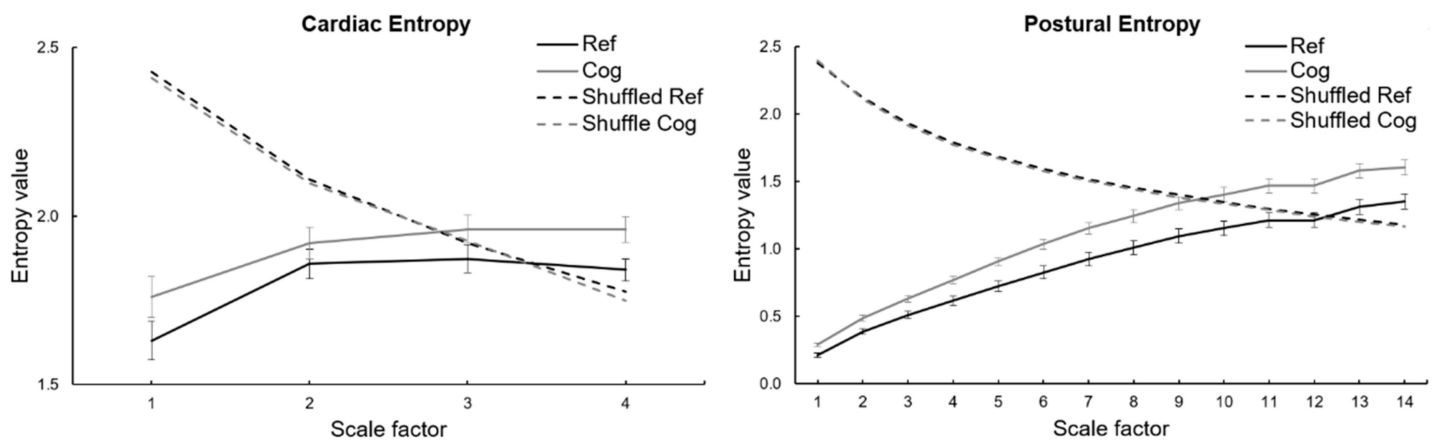

Figure 3. Refined composite multiscale entropy (RCMSE) analysis of RR interval time series (left) and center of pressure time series on anteroposterior axis (right) during reference (Ref) and cognitive (Cog) conditions. The RCMSE curves were obtained by connecting the group mean values of sample entropy for each scale. The error bars represent standard errors. The RCMSE curves for the surrogate shuffled time series are also presented.

\subsection{ROC Curves Analysis}

The ROC curves are shown in Figure 4, and the corresponding areas under the curves (AUC) and the Youden's indexes are reported in Table 2. The greatest AUC was obtained for entropy of both cardiac (0.67) and postural (0.72) time series, thus indicating that entropy showed a higher probability to assign a higher value to a positive instance than to a negative one.
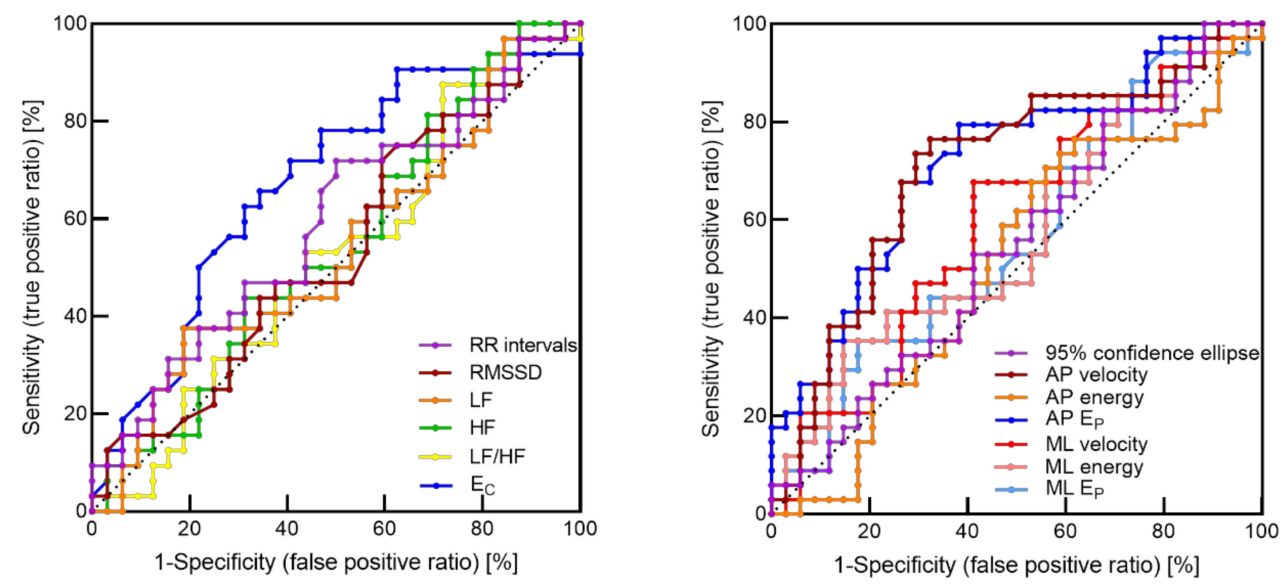

Figure 4. Receiver operating characteristic (ROC) curves (sensitivity vs 1-specificity) for cardiac (left) and postural (right) indices. RMSSD: root mean square of successive differences; LF: low frequency; HF: high frequency; $\mathrm{E}_{\mathrm{C}}$ : cardiac entropy index; $\mathrm{AP}$ : anteroposterior; $\mathrm{E}_{\mathrm{P}}$ : postural entropy index; ML: mediolateral. 
Table 2. Sensitivity analysis of cardiac and postural indices.

\begin{tabular}{ccc}
\hline Heart Rate Dynamics & $J$ & AUC \\
\hline RR intervals & 0.22 & 0.59 \\
RMSSD & 0.13 & 0.54 \\
LFs & 0.19 & 0.54 \\
HFs & 0.13 & 0.54 \\
LFs/HFs & 0.16 & 0.52 \\
E $_{C}$ & 0.31 & 0.67 \\
\hline Postural Dynamics & $J$ & AUC \\
\hline 95\% confidence ellipse $\left(\mathrm{mm}^{2}\right)$ & 0.15 & 0.55 \\
AP velocity & 0.44 & 0.71 \\
AP energy & 0.15 & 0.51 \\
AP E & 0.41 & 0.72 \\
ML velocity & 0.27 & 0.60 \\
ML energy & 0.21 & 0.67 \\
ML E & 0.18 & 0.56
\end{tabular}

$J$ : Youden's index; AUC: area under the ROC curve; RMSSD: root mean square of successive differences; LFs: low frequencies; $\mathrm{HFs}$ : high frequencies; $\mathrm{E}_{\mathrm{C}}$ : cardiac entropy index; $\mathrm{AP}$ : anteroposterior; $\mathrm{E}_{\mathrm{P}}$ : postural entropy index; ML: mediolateral.

\section{Discussion}

In this study we attempted to highlight the possible links between entropy measurements in two distinct neurophysiological networks and the systems complexity that probably facilitates the auto-organization and flexible adaptability in our healthy participants.

The main finding was that performing cognitive tasks resulted in a heightened entropy in heart rate and postural oscillations in young healthy people when compared to quiet conditions, as hypothesized. This may demonstrate that eliciting brain activity induced a remodeling in involuntary control networks, leading to a greater richness in signal information. This result is coherent with a great flexibility in our healthy young participants, which contrasts with a decline in entropy reported in older-aged individuals during a dual-task $[3,8]$. Both the elevation of entropy during cognitive tasks and the fact that two different neurophysiological systems behave in the same way represent original findings in the present study.

The link between central (cognitive) and peripheral regulations has been widely acknowledged. As a topic of growing interest, heart-brain interactions rely on a complex network of interconnected neural structures in the central autonomic network, whose functions are organized at the forebrain, brainstem, and spinal levels [37-41]. As shown by functional imaging, cortical and subcortical brain activities influence autonomic outflow to the periphery [42-45]. In our conditions, executive functions and associated prefrontal regions were involved during the imposed cognitive tasks. It is likely that the recruitment of brain regions reverberated throughout the autonomic outflow, as reflected in the heightened complexity revealed here by the RCMSE metrics in heart rate dynamics.

The rise in cardiac entropy is a marker of complex dynamics, which has been shown to reflect an underlying highly dimensional system with multiple interacting components associated with a high level of functionality [46,47]. Therefore, we can suggest that the observed increase in entropy during the cognitive tasks relies on remodeling and adaptability from the baseline, triggered by the recruitment and the interactions between brain components. This capacity to reorganize the control network in such a way that complexity is increased underscores a system's reserve that is not exhausted by any of our conditions [1]. This observation is in agreement with Costa et al. [1], who demonstrated that cardiac entropy (MSE) rose in healthy young people when facing diurnal challenges (waking period) that are absent during the night (sleep period). Cardiac entropy failed to increase comparatively in older-aged subjects. Other complexity metrics of HRV dynamics, such as fractal long-range properties in the temporal structure, provided additional evidence that cardiac complexity rises when the brain 
performs executive functions, which was reflected in clearer 1/f noise [48]. Yet, entropy metrics may provide greater reliability for analyzing complexity from short-term $\mathrm{HRV}$, because fractal properties are mainly dictated by power versus frequency characteristics of two dominant oscillators relying on vagal and sympathetic controls [49]. Hence, the "true fractal" component of the spectrum should be assessed only on frequencies $<0.04 \mathrm{~Hz}$, which requires long-lasting RR interval time series recordings [50]. Noticeably, RCMSE provided satisfactory results for the presence of a complex (1/f) system's behavior in our conditions (10 min recordings).

MSE has traditionally been computed to study the COP trajectory as an index of complexity in the neurophysiological control of posture, and a number of recommendations have been very useful in this domain [31]. The pre-processing of the COP signal in the present study (EMD filtering) is part of the cautious approach that is recommended. While it is usually reported that dual-tasking provides a decline in entropy among older-age individuals, we clearly show in this study that COP entropy rose (rather than dropped) in our young healthy participants. This highlights an adaptive capacity when recruiting cognitive functions and their related brain regions, which contrasts with the degraded $[3,8]$, but reversible [51], flexibility in older-aged dual-tasking.

It is not trivial to observe a similar behavior (the increase of entropy) in the present study both in relation to cardiovascular and postural control among our participants as a response to the cognitive task. These systems are markedly different; while the cardiac control relies on neurovisceral integration, the postural sway results from the somatosensory integration of exteroceptive and proprioceptive information. The rise in entropy therefore seems ubiquitous, and as such may reflect an adequate dynamic organization of neurophysiological control with improved interactions both within and between systems, whatever their neural structures.

Although the discovery of an increase in systems complexity in response to cognitive tasks is original in the present study, previous recent experiments have demonstrated that specific interventions may improve a degraded complexity. In humans, the capacity to restore a degraded postural complexity in aged people has been shown following mind-body interventions $[4,7,9]$. As well, walking arm-in-arm has recently been shown as an efficient way to restore walking complexity among older-aged individuals [52]. For years, degraded complexity markers (fractal or entropy metrics) in physiological signal outputs have been associated with impaired physiological control. The present study participates in the recent demonstrations of a heightened complexity marker indicating improved neurophysiological control.

\section{Conclusions}

By comparing quiet and cognitive task conditions, MSE-based metrics emphasize an adaptive systems capacity and a potential remodelling of cardiac and postural control systems under temporary states of cognitive tasks. The rise in entropy associated with cognitive functions, which contrasts with a decline reported in old people, illustrates improved interactions across brain regions and peripheral control loops, leading to a great richness in regulatory information. This demonstrates that the functional reserve capacity was not reached by our young healthy participants under our conditions. The issue of overwhelmed control systems in healthy young people confronted with cognitive tasks remained to be explored, through varying cognitive workloads or combining them with challenging emotions (e.g., stress), for example. It would be great to observe that whether, after heightening entropy in young people, more strenuous cognitive loads (with or without additional stressors) could push control systems to their adaptive limits, and whether this is reflected by a decline in entropy. It is unknown if the two distinct neurophysiological systems will keep demonstrating a similar behaviour when one faces such gradual challenges. With further study, even more credit could be gained towards entropy metrics and their capacity to faithfully reflect tight adjustments in complex physiological systems during gradual stimulations. 


\section{Limitations}

Despite appealing results, the present study was not without limitations. The number of participants might have been augmented, in particular the number of females offering the opportunity to explore sexual dimorphism, as noted elsewhere [53]. Regarding gender, it was noted that even a methodological choice for MSE may influence physiological interpretations due to sex-related differences in cardiovascular dynamics [30]. While we used a fixed tolerance $r$ at all scales in this study, an alternative method suggests adjusting the tolerance to the standard-deviation changes after coarse graining [30]. This might improve MSE estimation of heart rate and could be tested on the present data. It is presently unlikely that adopting an alternative (among many possible) usage of MSE could change the main conclusions of the present study; indeed, RCMSE on shuffle time series was computed here, clearly highlighting the distance from a random neurophysiological control and the capacity of RCMSE to distinguish quiet and cognitive task conditions (Figure 3). Finally, we have no explanation for the lack of change in ML entropy due to the cognitive task during postural regulation. Further studies are needed to explore the potential role of certain instances that could dominantly aggregate AP information, making complex AP regulations more responsive than ML.

Author Contributions: Conceptualization, E.B. and V.D.-A.; Methodology, E.B. and V.D.-A.; Software, E.B., L.M.A., P.G. and V.D.-A.; Validation, E.B., L.M.A., P.G. and V.D.-A.; Formal analysis, E.B.; Investigation, E.B.; Resources, E.B., L.M.A., P.G. and V.D.-A.; Data curation, E.B.; Writing-Original draft preparation, E.B., L.M.A., and V.D.-A.; Writing - Review and editing, E.B., L.M.A., P.G. and V.D.-A.

Funding: This research received no external funding.

Acknowledgments: The authors thank Yoel Kidane for reviewing the English manuscript.

Conflicts of Interest: The authors declare no conflict of interest.

\section{References}

1. Costa, M.; Goldberger, A.L.; Peng, C.-K. Multiscale entropy analysis of biological signals. Phys. Rev. E Stat. Nonlinear Soft Matter Phys. 2005, 71, 021906. [CrossRef] [PubMed]

2. Costa, M.; Goldberger, A.L.; Peng, C.-K. Multiscale entropy analysis of complex physiologic time series. Phys. Rev. Lett. 2002, 89, 68102. [CrossRef] [PubMed]

3. Kang, H.G.; Costa, M.D.; Priplata, A.A.; Starobinets, O.V.; Goldberger, A.L.; Peng, C.-K.; Kiely, D.K.; Cupples, L.A.; Lipsitz, L.A. Frailty and the degradation of complex balance dynamics during a dual-task protocol. J. Gerontol. A Biol. Sci. Med. Sci. 2009, 64, 1304-1311. [CrossRef] [PubMed]

4. Wayne, P.M.; Gow, B.J.; Costa, M.D.; Peng, C.-K.; Lipsitz, L.A.; Hausdorff, J.M.; Davis, R.B.; Walsh, J.N.; Lough, M.; Novak, V.; et al. Complexity-Based Measures Inform Effects of Tai Chi Training on Standing Postural Control: Cross-Sectional and Randomized Trial Studies. PLoS ONE 2014, 9, e114731. [CrossRef] [PubMed]

5. Richman, J.S.; Moorman, J.R. Physiological time-series analysis using approximate entropy and sample entropy. Am. J. Physiol. Heart Circ. Physiol. 2000, 278, H2039-H2049. [CrossRef] [PubMed]

6. Costa, M.; Priplata, A.A.; Lipsitz, L.A.; Wu, Z.; Huang, N.E.; Goldberger, A.L.; Peng, C.-K. Noise and poise: Enhancement of postural complexity in the elderly with a stochastic-resonance-based therapy. Europhys. Lett. 2007, 77, 68008. [CrossRef] [PubMed]

7. Wayne, P.M.; Manor, B.; Novak, V.; Costa, M.D.; Hausdorff, J.M.; Goldberger, A.L.; Ahn, A.C.; Yeh, G.Y.; Peng, C.-K.; Lough, M.; et al. A systems biology approach to studying Tai Chi, physiological complexity and healthy aging: design and rationale of a pragmatic randomized controlled trial. Contemp. Clin. Trials 2013, 34, 21-34. [CrossRef]

8. Manor, B.; Costa, M.D.; Hu, K.; Newton, E.; Starobinets, O.; Kang, H.G.; Peng, C.K.; Novak, V.; Lipsitz, L.A. Physiological complexity and system adaptability: Evidence from postural control dynamics of older adults. J. Appl. Physiol. 2010, 109, 1786-1791. [CrossRef] 
9. Manor, B.; Lipsitz, L.A.; Wayne, P.M.; Peng, C.-K.; Li, L. Complexity-based measures inform Tai Chi's impact on standing postural control in older adults with peripheral neuropathy. BMC Complement. Altern. Med. 2013, 13, 87. [CrossRef]

10. Busa, M.A.; Jones, S.L.; Hamill, J.; van Emmerik, R.E.A. Multiscale entropy identifies differences in complexity in postural control in women with multiple sclerosis. Gait Posture 2016, 45, 7-11. [CrossRef]

11. Gruber, A.H.; Busa, M.A.; Gorton Iii, G.E.; Van Emmerik, R.E.A.; Masso, P.D.; Hamill, J. Time-to-contact and multiscale entropy identify differences in postural control in adolescent idiopathic scoliosis. Gait Posture 2011, 34, 13-18. [CrossRef] [PubMed]

12. Tsai, C.-H.; Ma, H.-P.; Lin, Y.-T.; Hung, C.-S.; Hsieh, M.-C.; Chang, T.-Y.; Kuo, P.-H.; Lin, C.; Lo, M.-T.; Hsu, H.-H.; et al. Heart Rhythm Complexity Impairment in Patients with Pulmonary Hypertension. Sci. Rep. 2019, 9, 1-8. [CrossRef] [PubMed]

13. Liu, H.; Yang, Z.; Meng, F.; Huang, L.; Qu, W.; Hao, H.; Zhang, J.; Li, L. Chronic vagus nerve stimulation reverses heart rhythm complexity in patients with drug-resistant epilepsy: An assessment with multiscale entropy analysis. Epilepsy Behav. 2018, 83, 168-174. [CrossRef] [PubMed]

14. Silva, L.E.V.; Lataro, R.M.; Castania, J.A.; da Silva, C.A.A.; Valencia, J.F.; Murta, L.O.; Salgado, H.C.; Fazan, R.; Porta, A. Multiscale entropy analysis of heart rate variability in heart failure, hypertensive, and sinoaortic-denervated rats: classical and refined approaches. Am. J. Physiol. Regul. Integr. Comp. Physiol. 2016, 311, R150-R156. [CrossRef]

15. Silva, L.E.V.; Lataro, R.M.; Castania, J.A.; Silva, C.A.A.; Salgado, H.C.; Fazan, R.; Porta, A. Nonlinearities of heart rate variability in animal models of impaired cardiac control: contribution of different time scales. J. Appl. Physiol. 2017, 123, 344-351. [CrossRef]

16. Silva, L.E.V.; Silva, C.A.A.; Salgado, H.C.; Fazan, R. The role of sympathetic and vagal cardiac control on complexity of heart rate dynamics. Am. J. Physiol. Heart Circ. Physiol. 2017, 312, H469-H477. [CrossRef]

17. Marwaha, P.; Sunkaria, R.K. Exploring total cardiac variability in healthy and pathophysiological subjects using improved refined multiscale entropy. Med. Biol. Eng. Comput. 2017, 55, 191-205. [CrossRef]

18. O'Keeffe, C.; Taboada, L.P.; Feerick, N.; Gallagher, L.; Lynch, T.; Reilly, R.B. Complexity based measures of postural stability provide novel evidence of functional decline in fragile X premutation carriers. J. Neuroeng. Rehabil. 2019, 16, 87. [CrossRef]

19. Jiang, B.C.; Yang, W.-H.; Shieh, J.-S.; Fan, J.S.-Z.; Peng, C.-K. Entropy-based method for COP data analysis. Theor. Issues Ergon. Sci. 2013, 14, 227-246. [CrossRef]

20. Miyake, A.; Friedman, N.P.; Emerson, M.J.; Witzki, A.H.; Howerter, A.; Wager, T.D. The Unity and Diversity of Executive Functions and Their Contributions to Complex "Frontal Lobe" Tasks: A Latent Variable Analysis. Cogn. Psychol. 2000, 41, 49-100. [CrossRef]

21. Vendrell, P.; Junqué, C.; Pujol, J.; Jurado, M.A.; Molet, J.; Grafman, J. The role of prefrontal regions in the Stroop task. Neuropsychologia 1995, 33, 341-352. [CrossRef]

22. Stroop, J.R. Studies of interference in serial verbal reactions. J. Exp. Psychol. 1935, 18, 643-662. [CrossRef]

23. Burgess, P.W.; Shallice, T. The Hayling and Brixton Tests; Thames Valley Test Company: Bury St Edmunds, UK, 1997.

24. Gronwall, D.M. Paced auditory serial-addition task: a measure of recovery from concussion. Percept. Mot. Skills 1977, 44, 367-373. [CrossRef] [PubMed]

25. Stokholm, J.; Jørgensen, K.; Vogel, A. Performances on five verbal fluency tests in a healthy, elderly Danish sample. Neuropsychol. Dev. Cogn. B Aging. Neuropsychol. Cogn. 2013, 20, 22-33. [CrossRef] [PubMed]

26. Camm, A.J.; Malik, M.; Bigger, J.T.; Breithardt, G.; Cerutti, S.; Cohen, R.J.; Lombardi, F. Heart rate variability. Standards of measurement, physiological interpretation, and clinical use. Task Force of the European Society of Cardiology and the North American Society of Pacing and Electrophysiology. Eur. Heart J. 1996, 17, 354-381.

27. Wu, S.-D.; Wu, C.-W.; Lin, S.-G.; Lee, K.-Y.; Peng, C.-K. Analysis of complex time series using refined composite multiscale entropy. Phys. Lett. A 2014, 378, 1369-1374. [CrossRef]

28. Humeau-Heurtier, A. The Multiscale Entropy Algorithm and Its Variants: A Review. Entropy 2015, 17, 3110-3123. [CrossRef] 
29. Wu, S.-D.; Wu, C.-W.; Lin, S.-G.; Wang, C.-C.; Lee, K.-Y. Time Series Analysis Using Composite Multiscale Entropy. Entropy 2013, 15, 1069-1084. [CrossRef]

30. Castiglioni, P.; Coruzzi, P.; Bini, M.; Parati, G.; Faini, A. Multiscale Sample Entropy of Cardiovascular Signals: Does the Choice between Fixed- or Varying-Tolerance among Scales Influence Its Evaluation and Interpretation? Entropy 2017, 19, 590. [CrossRef]

31. Gow, B.J.; Peng, C.-K.; Wayne, P.M.; Ahn, A.C. Multiscale Entropy Analysis of Center-of-Pressure Dynamics in Human Postural Control: Methodological Considerations. Entropy 2015, 17, 7926-7947. [CrossRef]

32. Huang, N.E.; Shen, Z.; Long, S.R.; Wu, M.C.; Shih, H.H.; Zheng, Q.; Yen, N.-C.; Tung, C.C.; Liu, H.H. The empirical mode decomposition and the Hilbert spectrum for nonlinear and non-stationary time series analysis. Proc. R. Soc. Lond. A Math. Phys. Eng. Sci. 1998, 454, 903-995. [CrossRef]

33. Metz, C.E. Basic principles of ROC analysis. Semin. Nucl. Med. 1978, 8, 283-298. [CrossRef]

34. Hanley, J.A.; McNeil, B.J. The meaning and use of the area under a receiver operating characteristic (ROC) curve. Radiology 1982, 143, 29-36. [CrossRef] [PubMed]

35. Zhou, J.; Habtemariam, D.; Iloputaife, I.; Lipsitz, L.A.; Manor, B. The Complexity of Standing Postural Sway Associates with Future Falls in Community-Dwelling Older Adults: The MOBILIZE Boston Study. Sci. Rep. 2017, 7, 2924. [CrossRef] [PubMed]

36. Błaszczyk, J.W.; Klonowski, W. Postural stability and fractal dynamics. Acta Neurobiol. Exp. 2001, 61, $105-112$.

37. Benarroch, E.E. The central autonomic network: functional organization, dysfunction, and perspective. Mayo Clin. Proc. 1993, 68, 988-1001. [CrossRef]

38. Thayer, J.F.; Ahs, F.; Fredrikson, M.; Sollers, J.J.; Wager, T.D. A meta-analysis of heart rate variability and neuroimaging studies: implications for heart rate variability as a marker of stress and health. Neurosci. Biobehav. Rev. 2012, 36, 747-756. [CrossRef]

39. Thome, J.; Densmore, M.; Frewen, P.A.; McKinnon, M.C.; Théberge, J.; Nicholson, A.A.; Koenig, J.; Thayer, J.F.; Lanius, R.A. Desynchronization of autonomic response and central autonomic network connectivity in posttraumatic stress disorder. Hum. Brain Mapp. 2017, 38, 27-40. [CrossRef]

40. Beissner, F.; Meissner, K.; Bär, K.-J.; Napadow, V. The autonomic brain: an activation likelihood estimation meta-analysis for central processing of autonomic function. J. Neurosci. 2013, 33, 10503-10511. [CrossRef]

41. Thayer, J.F.; Lane, R.D. Claude Bernard and the heart-brain connection: further elaboration of a model of neurovisceral integration. Neurosci. Biobehav. Rev. 2009, 33, 81-88. [CrossRef]

42. Allen, B.; Jennings, J.R.; Gianaros, P.J.; Thayer, J.F.; Manuck, S.B. Resting high-frequency heart rate variability is related to resting brain perfusion. Psychophysiology 2015, 52, 277-287. [CrossRef] [PubMed]

43. Gianaros, P.J.; Wager, T.D. Brain-Body Pathways Linking Psychological Stress and Physical Health. Curr. Dir. Psychol. Sci. 2015, 24, 313-321. [CrossRef] [PubMed]

44. Sakaki, M.; Yoo, H.J.; Nga, L.; Lee, T.-H.; Thayer, J.F.; Mather, M. Heart rate variability is associated with amygdala functional connectivity with MPFC across younger and older adults. Neuroimage 2016, 139, 44-52. [CrossRef] [PubMed]

45. Lane, R.D.; McRae, K.; Reiman, E.M.; Chen, K.; Ahern, G.L.; Thayer, J.F. Neural correlates of heart rate variability during emotion. Neuroimage 2009, 44, 213-222. [CrossRef]

46. Lipsitz, L.A. Dynamics of stability: The physiologic basis of functional health and frailty. J. Gerontol. A Biol. Sci. Med. Sci. 2002, 57, B115-B125. [CrossRef]

47. Delignieres, D.; Marmelat, V. Fractal fluctuations and complexity: current debates and future challenges. Crit. Rev. Biomed. Eng. 2012, 40, 485-500. [CrossRef]

48. Hoshikawa, Y.; Yamamoto, Y. Effects of Stroop color-word conflict test on the autonomic nervous system responses. Am. J. Physiol. 1997, 272, H1113-H1121. [CrossRef]

49. Francis, D.P.; Willson, K.; Georgiadou, P.; Wensel, R.; Davies, L.C.; Coats, A.; Piepoli, M. Physiological basis of fractal complexity properties of heart rate variability in man. J. Physiol. 2002, 542, 619-629. [CrossRef]

50. Castiglioni, P. Commentary: Decomposition of Heart Rate Variability Spectrum into a Power-Law Function and a Residual Spectrum. Front. Cardiovasc. Med. 2018, 5, 94. [CrossRef]

51. Chen, M.-S.; Jiang, B.C. Resistance training exercise program for intervention to enhance gait function in elderly chronically ill patients: Multivariate multiscale entropy for center of pressure signal analysis. Comput. Math. Methods Med. 2014, 2014, 471356. [CrossRef] 
52. Almurad, Z.M.H.; Roume, C.; Blain, H.; Delignières, D. Complexity Matching: Restoring the Complexity of Locomotion in Older People Through Arm-in-Arm Walking. Front. Physiol. 2018, 9, 1766. [CrossRef] [PubMed]

53. Young, H.; Benton, D. We should be using nonlinear indices when relating heart-rate dynamics to cognition and mood. Sci. Rep. 2015, 5, 16619. [CrossRef] [PubMed]

(C) 2019 by the authors. Licensee MDPI, Basel, Switzerland. This article is an open access article distributed under the terms and conditions of the Creative Commons Attribution (CC BY) license (http://creativecommons.org/licenses/by/4.0/). 